\title{
REVISIÓN
}

\section{Gliomas de tallo cerebral; generalidades de diagnóstico, tratamiento y pronóstico}

\author{
Pamela Peiro Osuna,* Agustín Alentorn,** Alán Hernández-Hernández,* Ignacio Reyes-Moreno,*** \\ Guillermo A. Gutiérrez Aceves, ${ }^{* * *, * * * *}$ Alberto González-Aguilar*,***,***** \\ * Departamento de Urgencias. Instituto Nacional de Neurología y Neurocirugía (INNN). \\ ** Servicio de Neurooncología. Hospital de la Pitié-Salpêtrière, París, Francia \\ *** Centro Neurológico ABC Santa Fe, Centro Médico ABC, México. \\ **** Servicio de Radiocirugía. INNN. \\ ***** Clínica de Neurooncología. INNN.
}

\begin{abstract}
RESUMEN
Los gliomas de tallo cerebral son poco comunes, en los adultos representan 1-2\% de los gliomas intracraneales. En los adultos predomina un fenotipo de bajo grado, una característica que probablemente explica su mejor pronóstico en comparación con los niños. Debido a que la biopsia se realiza con poca frecuencia, se ha propuesto una clasificación basada en los aspectos radiológicos en resonancia magnética para establecer estrategias de tratamiento y determinar el resultado: 1. Intrínseco difuso "de bajo grado", 2. Glioma de tallo "maligno" con reforzamiento focal (GTMRF), 3. Exofítico y 4. Gliomas focales tectales. A pesar de los importantes avances en neurorradiología tales como espectroscopia de resonancia magnética multivoxel, tensor de difusión y perfusión, una clasificación radiológica pura sigue siendo imperfecta en ausencia de un diagnóstico histológico. Mientras que la biopsia puede ser razonablemente evitada en las formas difusas, es necesaria una prueba histológica en muchas lesiones del tallo cerebral cuando se evidencian lesiones captantes de contraste debido a la gran variedad de diagnósticos diferenciales en los adultos. La radioterapia es el tratamiento estándar para los gliomas difusos intrínsecos de "bajo grado" de tallo cerebral en los adultos (la media de supervivencia es de cinco años). En los gliomas "malignos" de tallo con reforzamiento focal, la radioterapia es el tratamiento estándar y el posible beneficio de la radioterapia y la quimioterapia combinada (temozolamida $u$ otros agentes) no se ha evaluado a fondo en adultos. El papel de las terapias con antiangiogénicos en gliomas de tallo cerebral está todavía por definirse. Una mejor comprensión de la biología de estos tumores es esencial para identificar subgrupos homogéneos y mejorar la terapia.
\end{abstract}

Palabras clave: Glioma, tallo cerebral, intracraneal, radioterapia.

\section{ABSTRACT}

Brainstem gliomas are uncommon, in adults they account for 1-2\% of intracranial gliomas. In adults, a low-grade phenotype predominates, a feature that probably accounts for its better prognosis compared to children. Because biopsy is performed infrequently, a classification based on radiological aspects in MRI has been proposed to establish treatment strategies and determine the outcome: 1. Intrinsic diffuse "low-grade". 2. Brainstem glioma "malignant" with focal reinforcement. 3. Exophytic. 4. Focal tectal gliomas. Despite important advances in neuroradiology such as multivoxel magnetic resonance spectroscopy, diffusion tensor and perfusion, a pure radiological classification remains imperfect in the absence of a histological diagnosis. While biopsy can be reasonably avoided in diffuse forms, a histological test is required in many brainstem lesions when contrast-enhancing lesions are evident because of the wide variety of differential diagnoses in adults. Radiation therapy is the standard treatment for intrinsic diffuse brainstem gliomas "low-grade" in adults (mean survival rate is 5 years). In malignant stem gliomas with focal reinforcement, radiation therapy is the standard treatment and the possible benefit of radiotherapy and combination chemotherapy (temozolamide or other agents) has not been thoroughly evaluated in adults. The role of anti-angiogenic therapies in brainstem gliomas remains to be defined. A better understanding of the biology of these tumors is of great importance in order to identify homogeneous subgroups and improve therapy.

Key words: Glioma, brain stem, intracranial, radiotherapy.

\section{INTRODUCCIÓN}

Los tumores de tallo cerebral se definen como lesiones que ocurren en el mesencéfalo, puente o bulbo raquídeo. Esta definición excluye a los pacientes con tumores que se originan en el tálamo y el hipotálamo o las lesiones que se originan en el cerebelo, pedúnculos cerebelosos o en la médula espinal cervical superior. Su extensión se considera "focal" cuando ocupan menos de $50 \%$ del diámetro del tallo cerebral y "difusa" cuando la lesión está mal delimitada con una extensión de más de 50\% del diámetro del 
tallo cerebral. ${ }^{1-3}$ Los gliomas de tallo cerebral son los tumores más frecuentes de la región. Una clara distribución por edad bimodal sostiene una distinción de los gliomas del tallo cerebral entre niños y adultos. En contraste con la población pediátrica, en la que los gliomas del tallo cerebral representan hasta $20 \%$ de los tumores cerebrales y muestran una evolución desfavorable y no homogénea, los gliomas del tallo cerebral en adultos son raros (1-2\% de los gliomas cerebrales en adultos) y heterogéneos con patrón radiológico diferente y pronóstico variable. ${ }^{4,5}$ En este trabajo se revisa la literatura sobre los gliomas de tallo cerebral en adultos, reconociendo los problemas que encontramos para extraer datos comparables en muchos artículos (heterogeneidad de los términos utilizados, las dificultades para evaluar el tratamiento y el curso de los pacientes). El hecho de que las biopsias rara vez se realizan, no permite un análisis basado sólo en criterios histológicos, por ende se utilizó una clasificación radiológica fundamentada por resonancia magnética (IRM) considerando cuatro grandes grupos: 1. glioma intrínseco difuso o difusamente infiltrante "bajo grado" del tallo cerebral (GTCID), 2. glioma de tallo maligno con reforzamiento focal (GTMRF), 3. gliomas focales tectales y 4 . gliomas exofíticos. ${ }^{4,6}$

\section{Glioma del tallo cerebral intrínseco difuso (GTCID) “bajo grado”}

\section{Definición}

Este tipo de tumor se define radiológicamente como una lesión mal delimitada que no refuerza el medio de contraste y que afecta más de $50 \%$ del diámetro del tallo cerebral. ${ }^{2,3}$ Aproximadamente en $80 \%$ de los pacientes se ve afectado más de un nivel del tallo cerebral (mesencéfalo, puente, bulbo raquídeo) en el momento del diagnóstico. Las estructuras pontinomedulares son la localización más frecuente en la población adulta. Durante el curso clínico de la lesión puede infiltrarse en otras regiones, tales como la unión cervicomedular, el cerebelo, tálamo y los ganglios basales. Por lo general, el GTCID es un tumor de bajo grado al inicio, pero evoluciona hacia un tumor de grado más alto que se manifiesta por el desarrollo de lesiones captantes de contraste, en IRM se describe en $27 \%$ de los pacientes en dos series y el crecimiento incesante y sin cambios se ha reportado en $23 \%$ (Figura 1) ${ }^{4,7}$ En la actualidad, todavía no se sabe si esta supuesta transformación anaplásica es la evolución natural a largo plazo para todos los GTCID.

\section{Epidemiología}

El GTCID suele aparecer en adultos jóvenes entre 20 y 50 años de edad (la edad media es de 34 años al momento del diagnóstico). ${ }^{5,8,9}$ Representa el tipo más frecuente de GTC en los adultos, lo que equivale a $45-50 \%$ de los casos. ${ }^{4,10,11}$

\section{Patología}

Rara vez se toma una biopsia en el glioma intrínseco difuso típico en adultos, pero cuando se lleva a cabo, se observa una histología benigna (glioma de grado II) en hasta $80 \%$ de los casos, mientras que en los niños un glioma de grado IV es el fenotipo más frecuente reportado en $50-60 \%$ de los casos. ${ }^{10}$ Esta característica explica probablemente el mejor pronóstico de la forma adulta. 4,5,8,12 Sin embargo, los pacientes adultos en ocasiones presentan un rápido crecimiento del tumor similar a GTCID en la población pediátrica. ${ }^{4,13}$
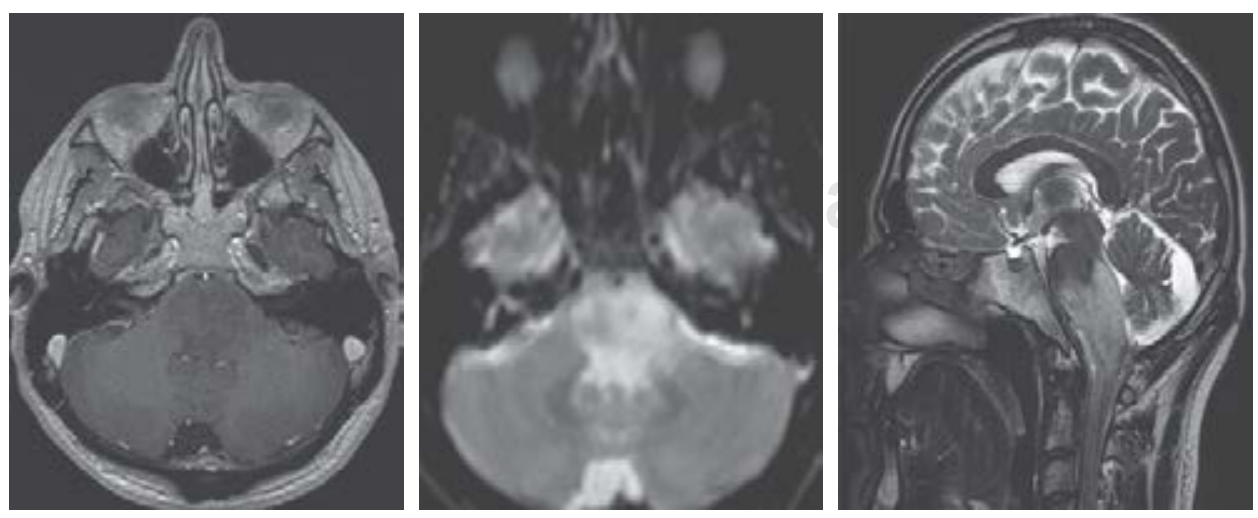

Figura 1.

IRM axial y sagital. Lesión hipointensa en T1 sin captación de contraste, pero en el estudio de difusión y el corte sagital se observa el carácter infiltrativo y diseminado de los gliomas de Tallo. 
Histológicamente, los gliomas del tallo en adultos pueden tener una apariencia astrocítica, oligodendroglial o mixta, con tumores astrocíticos caracterizados además como pilocíticos o difusamente infiltrativos. Un análisis retrospectivo de una institución italiana de 21 pacientes con enfermedad confirmada histológicamente identificó dos astrocitomas pilocíticos, nueve astrocitomas de bajo grado, ocho astrocitomas anaplásicos y un glioblastoma. Un análisis retrospectivo de MD Anderson que incluyó 98 casos con histología, identificó 28 glioblastomas, 43 astrocitomas anaplásicos, 15 astrocitomas difusos y 11 gliomas no especificados. ${ }^{14}$ Según el conocimiento actual, parece que la mayoría de los gliomas del tallo cerebral en adultos pueden estratificarse en uno de dos grupos, según si el tumor tiene una mutación de IDH o una mutación de H3F3A. ${ }^{14}$ Se espera que un mejor conocimiento del perfil de las alteraciones moleculares en el tejido de los tumores ayude a clasificar y predecir el comportamiento del tumor, como se ha demostrado en los gliomas supratentoriales.

\section{Características clínicas}

Por lo regular, los gliomas de tallo cerebral en los adultos se detectan en pacientes con buen estado funcional de Karnofsky (KPS) $>80 \%$ en el momento del diagnóstico. Los síntomas y signos clínicos están estrechamente vinculados a la localización del tumor. Las alteraciones visuales, disfunción de nervios craneales, debilidad en las extremidades y trastornos de la marcha son los principales síntomas en la mayoría de los casos. 4,8,11,12 Generalmente, una combinación de disfunción del nervio craneal con signos de tractos o vías largas se considera sugerente de lesión cerebral. Los pacientes a menudo muestran historia de parálisis facial de larga duración que a veces se asocia a mioquimia facial o espasmo hemifacial. ${ }^{15} \mathrm{En}$ la exploración, la debilidad piramidal, signos cerebelosos o la participación de otros nervios craneales (V, VI, VIII, parálisis del paladar) también son comunes.

Presentaciones poco comunes incluyen ronquidos asociados al curso de ondina (hipertrofia alveolar primaria) en el caso de glioma bulbo raquídeo, hiperventilación neurogénica central, temblor de la lengua, hemiparkinsonismo, auras visuales migrañosas, así como presentaciones pseudomiasténicas. ${ }^{16-21}$

Otras manifestaciones clínicas tales como dolor de cabeza y aumento de la presión intracraneal pueden aparecer en el inicio cuando el tumor obstruye el drenaje de líquido cefalorraquídeo causando hidrocefalia, las cuales se reportan en $23 \%$ de los pacientes. ${ }^{8}$

\section{Imágenes}

En la actualidad la resonancia magnética (IRM) cerebral es la herramienta radiológica más útil para diagnosticar lesiones del tallo cerebral. Por lo general, revela un agrandamiento difuso de tallo cerebral con áreas hiperintensas en secuencias potenciadas en T2 y señales hipointensas en las imágenes potenciadas en T1. Los tumores son difusamente infiltrantes y no captan contraste después de la administración de gadolinio. ${ }^{22}$ Las lesiones pueden estar limitadas a un segmento de tallo cerebral (mesencéfalo, protuberancia y bulbo raquídeo) o se infiltran en otros segmentos del tallo cerebral o regiones adyacentes, como el cerebelo o el tálamo. En una serie de casos, el puente se vio afectado en $64 \%$ de los pacientes seguido de la médula en $51 \%$ y el mesencéfalo en $43 \%{ }^{4}$ Como grupo, los GTCID implican las estructuras pontinomedulares en $80 \%$ de los casos. ${ }^{12}$

La espectroscopia de resonancia magnética (ERM) es una herramienta complementaria utilizada en el diagnóstico diferencial de lesiones en tallo cerebral. Sin embargo, en comparación con su aplicación en lesiones supratentoriales, el uso de la ERM es mucho más limitado en el tallo cerebral, debido a dificultades técnicas relacionadas con el pequeño tamaño de las estructuras anatómicas y la proximidad de los huesos y el tejido graso de la base del cráneo. Actualmente la ERM de un solo vóxel es la técnica utilizada para estudiar las lesiones difusas del tallo cerebral, en especial en lesiones protuberanciales mayores de $2 \mathrm{~cm}$. Recientemente, la aplicación de la ERM 3T multivóxel ha mejorado la resolución espacial de esta técnica cuando los tiempos usados de adquisición son de 12 a 15 minutos. ${ }^{22}$ Para las lesiones en bulbo raquídeo el papel de la ERM aún está limitado (Figura 2).

En un estudio retrospectivo, nueve pacientes adultos que sufren de gliomas del tallo cerebral (confirmado histológicamente en cuatro casos) fueron evaluados con la ERM de un solo vóxel antes del tratamiento. ${ }^{8}$ Todos los pacientes mostraron una elevación en la relación de colina/ $\mathrm{N}$-acetil aspartato (relación Cho/NAA) de 1.08-3.32 (normal 0.6-0.8), una elevación de la relación Cho/creatina (ocho pacientes) y en la relación creatina/NAA debido a la pérdida de la señal de NAA. Por lo tanto, los cambios de la ERM parecen similares a los observados en los gliomas supratentoriales. En población pediátrica, la ERM se ha utilizado para detectar la progresión (disminución de NAA, elevación de Cho y disminución de la relación de NAA/Co y $\mathrm{Cr} / \mathrm{Cho}$ ) radiológica o deterioro clínico. ${ }^{23}$ La experiencia en niños también 


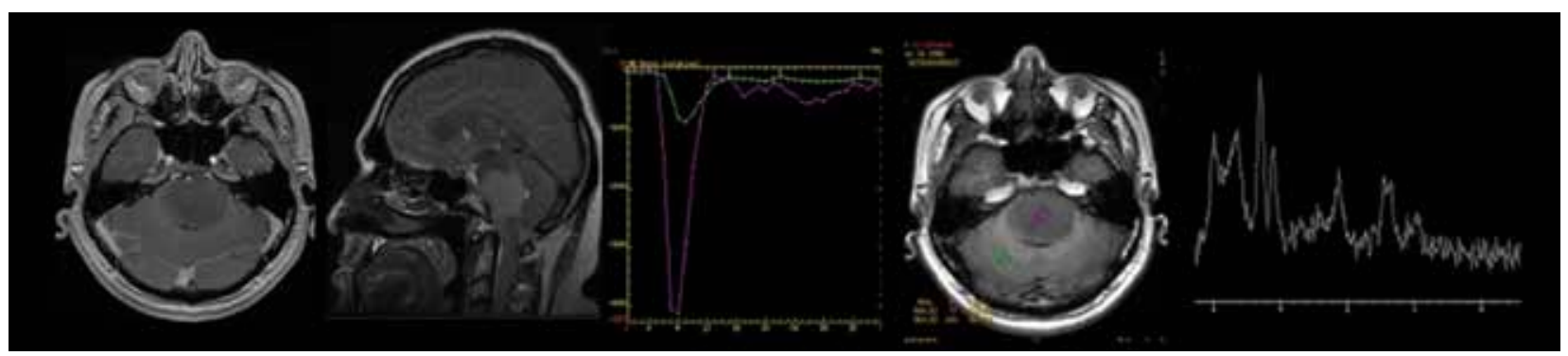

Figura 2. IRM contrastada con perfusión y espectroscopia. El estudio de perfusión presenta incremento significativo de la perfusión intratumoral y la espectroscopia muestra aumento de la colina e inversión de la relación Colina/N Acetil Aspartato.

sugiere que la ERM puede contribuir al diagnóstico diferencial con enfermedades infecciosas y desmielinizantes. ${ }^{24}$

El tensor de difusión (DTI) y la tractografía también pueden ayudar a diferenciar el glioma difuso de tallo cerebral (fibras desviadas) de la enfermedad desmielinizante (falta de distorsión). ${ }^{25}$

\section{Principios de tratamiento}

\section{Tratamiento sintomático}

Los glucocorticoides se administran frecuentemente a pacientes con tumores del tallo cerebral. Al igual que en los gliomas supratentoriales, sus efectos beneficiosos se atribuyen a una reducción del edema asociado al tumor, mejorando los síntomas relacionados con la compresión del tracto largo de las fibras blancas. ${ }^{26}$ A pesar de su amplio uso en pacientes con GTC, se carece de revisiones sistemáticas o metaanálisis al respecto. En una serie retrospectiva, los glucocorticoides se administraron a $82(73 \%)$ pacientes con GTC durante la radioterapia y la mitad de ellos presentan dependencia a largo plazo para el alivio sintomático con múltiples efectos secundarios como miopatía, gastritis, osteonecrosis y psicosis. ${ }^{12}$ Los glucocorticoides deben usarse con moderación, a la dosis más baja necesaria para evitar estas complicaciones. Los pacientes con dificultades para deglutir pueden requerir gastrostomía percutánea como esofagogastrostomía para garantizar los requerimientos nutricionales diarios.

\section{Cirugía}

La resección de GTCID sigue siendo un reto por el hecho de las secuelas neurológicas que pueden generarse y la mínima cantidad de resección que se obtiene, un abordaje quirúrgico sólo está justificado en el caso de la colocación de una derivación para el alivio de síntomas. ${ }^{27,28}$ En la práctica, el papel de la cirugía se limita a la biopsia cuya indicación sigue siendo controvertida. Un estudio prospectivo incluyó 46 pacientes adultos con lesiones de tallo cerebral comparado con el diagnóstico preoperatorio evaluado por la IRM convencional contra el resultado histológico obtenido por biopsia estereotáctica. ${ }^{29}$ Aunque los gliomas intrínsecos difusos no fueron separados de las demás formas histológicas, los autores observaron que en aproximadamente $30 \%$ de los casos no hubo correlación histológica ni radiológica. La morbilidad perioperatoria fue de $4 \%$ y no se suscitó ninguna muerte relacionada con el procedimiento. Se concluyó que la biopsia estereotáctica guiada era relativamente segura y que debe recomendarse un diagnóstico preciso en todos los casos y no sólo cuando las características clínicas o de imágenes atípicas sugieran una lesión no neoplásica. En una cohorte alemana de 110 pacientes adultos se describió la baja sensibilidad de la IRM en la diferenciación del glioma de bajo grado (GBG) del alto grado con una sensibilidad de 41 y $21 \%$ respectivamente. ${ }^{30}$ Por el contrario, otros equipos consideran que los pacientes que muestran la imagen radiológica "clásica" de una lesión no captante infiltrante difusa no requieren biopsia, debido a una supuesta relación desfavorable riesgo/beneficio de esta última. ${ }^{4}$

En un reporte de casos de 96 pacientes con hallazgos histopatológicos de lesiones intrínsecas de tallo cerebral el efecto diagnóstico con biopsia estereotáctica en estas lesiones fue mayor en pacientes con lesiones focales o captantes de contraste por IRM que las lesiones difusas. ${ }^{31}$

Un abordaje quirúrgico agresivo en las lesiones intrínsecas mejora la tasa de supervivencia libre de progresión en $45 \%$ y la supervivencia global en $61 \%$ 
en cinco años, presentándose complicaciones con poca frecuencia. ${ }^{32}$

\section{Radioterapia}

La radioterapia fraccionada es el tratamiento estándar para GTCID en adultos y puede mejorar o estabilizar a los pacientes por años, logrando una supervivencia libre de progresión de hasta 7.3 años. ${ }^{7,11}$ La dosis media de radioterapia convencional es 50-55 Gy usando fracciones de 1.8-2 Gy. La mejoría clínica se produce en la mayoría de los pacientes, pero hay discrepancia sustancial entre la respuesta clínica y radiológica. En una serie de adultos que incluyó 22 (46\%) pacientes con GTCID, la radioterapia mejoró el estado clínico en $61 \%$, mientras que la respuesta radiológica parcial (disminución de 50\% en IRM T2 en la sección transversal axial mayor) se observó en sólo $19 \%{ }^{4}$

El momento óptimo de tratamiento sigue siendo desconocido. De hecho, algunos pacientes pueden tener síntomas leves durante largos periodos sin ningún tipo de tratamiento, lo que sugiere que en los gliomas supratentoriales de grado bajo la radioterapia puede a veces diferirse hasta que se confirme evidencia clara de la progresión del tumor sintomático. ${ }^{8}$

\section{Quimioterapia}

Los datos sobre la quimioterapia son muy escasos. En una serie de 34 pacientes, incluyendo todos los grupos de tumores de tallo cerebral (50\% GTCID), 22 pacientes fueron tratados con radioterapia conformal (48-58 Gy en fracciones de 1.8 Gy) asociados a la quimioterapia concomitante con TMZ inicial de 75 $\mathrm{mg} / \mathrm{m}^{2} /$ día y $200 \mathrm{mg} / \mathrm{m}^{2} /$ día 5 días/meses después de la radioterapia similar al régimen de Roger Stupp que se utiliza en glioblastomas supratentoriales. ${ }^{8}$ La tasa de supervivencia libre de progresión del tumor fue de 10 meses y la supervivencia global fue de 59 meses (todos los pacientes incluidos). Por desgracia, el hecho de que diferentes tipos de gliomas de tallo cerebral (en particular tumores de bajo y alto grado) se analizaron en conjunto, afecta seriamente la interpretación de estos datos y se desconoce si la quimioterapia concomitante/adyuvante tiene lugar en el tratamiento de forma intrínseca difusa.

En otro estudio reciente que incluyó 12 pacientes con glioma de tallo difuso recibieron 54 Gy de radioterapia conformal durante seis semanas con fracciones 1.8 Gy concomitante con temozolamida (TMZ) a dosis de TMZ oral diaria de $75 \mathrm{mg} / \mathrm{m}^{2} /$ día. Cuatro semanas después de la radioterapia, todos los pacientes recibieron seis ciclos de TMZ. En el primer ciclo se dio la TMZ a dosis de $150 \mathrm{mg} / \mathrm{m}^{2}$, seguida de $200 \mathrm{mg} / \mathrm{m}^{2}$ para el resto de los ciclos. La tasa general de beneficio clínico fue de $75 \%$. La tasa de supervivencia libre de progresión fue de $75 \%$ a los seis meses y $50 \%$ a un año. La tasa de supervivencia global al año fue $75 \%$. Respecto a los criterios de RANO (Revised Assessment in Neuro-Oncology) 8.3\% presentó respuesta completa, $50 \%$ respuesta parcial, $16.7 \%$ enfermedad estable y $25 \%$ progresión de la enfermedad. ${ }^{33}$

Un estudio retrospectivo evaluó la quimioterapia con varios agentes (nitrosureas, carboplatino, liste) en la recidiva después de la radioterapia en 14 pacientes y se observó un modesto $7 \%$ de la tasa de respuesta radiológica, pero la mejoría clínica se apreció en 15\% (Guillamo).

El bevacizumab se ha utilizado en GTCID de manera recurrente en pacientes adultos tras el fracaso de la radioterapia y temozolomida (TMZ). ${ }^{34,35}$ Los autores informaron una mejoría clínica significativa y una respuesta radiológica sostenida después de 12 meses de tratamiento. En los niños este régimen ha sido ineficaz en la recurrencia en un estudio de fase II. ${ }^{36}$

Por lo tanto, la eficacia de la quimioterapia en GTCID en adulto permanece sin probarse y la quimioterapia adyuvante no se recomienda actualmente. Por otra parte, la eficacia de la quimioterapia en la recaída es incierta, aunque puede beneficiar a algunos pacientes, a veces con una tajante mejoría clínica-radiológica, más a menudo con una mejora clínica aislada en contraste con la falta de respuesta radiológica.

\section{Pronóstico y seguimiento}

Raras ocasiones los adultos tienen una forma intrínseca difusa severa similar a la de los niños, es decir que corresponde a un glioma maligno y comparten un pronóstico similar (alrededor de un año), el patrón de crecimiento de la forma intrínseca clásica "bajo grado" en adultos es lento. La media de supervivencia de los GTCID en adultos oscila entre 4.75 y 7.3 años (en comparación con menos de un año en los niños), como se muestra en tres estudios retrospectivos europeos y estadounidenses recientes enfocados en GTCID en adultos. ${ }^{4,5,12,37}$

El patrón radiológico de evolución normal es una infiltración lenta y progresiva de todo el tallo cerebral que tiene lugar durante años. A veces, este patrón de progresión lenta es suplantado por el rápido desarrollo de una lesión captante de contraste dentro de las áreas 
infiltradas difusas, lo que sugiere la transformación de glioma de alto grado. La diseminación leptomeníngea se manifiesta por lesiones regionales y/o distantes en etapas avanzadas de la enfermedad, comúnmente asociadas a deterioro clínico. Con el tiempo, los pacientes desarrollan cuadriplejia, disartria severa, disfagia y múltiples parálisis de los nervios craneales, que a veces culminan en un síndrome de "enclaustramiento". La causa inmediata de la muerte es a menudo una complicación sistémica (embolia pulmonar, neumonía por aspiración, entre otras).

No hay factores pronósticos que hayan sido identificados en los GTCID en adultos. La literatura disponible no puede interpretarse con fiabilidad porque los datos a menudo mezclan adultos y niños, así como diversos subtipos de gliomas de tallo cerebral. Se sospecha de factores pronósticos peyorativos que incluyen el aumento de la edad al momento del diagnóstico ( $>45$ años) y la localización del tumor (pontino difuso), la raza (no caucásicos) y el tipo histológico. ${ }^{9,12}$

\section{Glioma de tallo “maligno” con reforzamiento focal (GTMRF)}

\section{Epidemiología}

El glioma maligno representa alrededor de 30\% de los tumores del tallo cerebral en adultos. ${ }^{7,10} \mathrm{En}$ comparación con los GTCID de bajo grado, usualmente éste aparece en pacientes mayores de 40 años de edad. ${ }^{4,5}$

\section{Patología}

El examen histológico es mucho más frecuente en GTMRF en comparación con el GTCID de bajo grado, debido a que el patrón de resonancia magnética no es específico en estos pacientes y las biopsias se toman más a menudo para confirmar el diagnóstico. Éstos corresponden principalmente a los astrocitomas anaplásicos y glioblastomas. Guillamo et al. reportaron 14 gliomas malignos (12 astrocitomas anaplásicos y dos oligodendrogliomas anaplásicos). ${ }^{4}$ Hay pocos datos que evalúan las posibles diferencias histológicas entre gliomas malignos de tallo cerebral y gliomas malignos supratentoriales, pero no hubo diferencias notables. ${ }^{38}$

\section{Las características clínicas}

La evolución clínica es subaguda, consta de parálisis de los nervios craneales, visión borrosa y signos piramidales que ocasionan rápidamente un estado funcional alterado. La presentación de los síntomas depende de la localización de la lesión. En comparación con los pacientes de GTCID, los pacientes con GTMRF se encuentran en peores condiciones clínicas y tienen una evolución mucho más breve de los síntomas antes del diagnóstico. ${ }^{12,39}$

\section{Imágenes}

Por lo regular, los GTMRF se manifiestan como masas captantes de contraste rodeadas por un área de edema. El realce del contraste se encuentra en $100 \%$ de
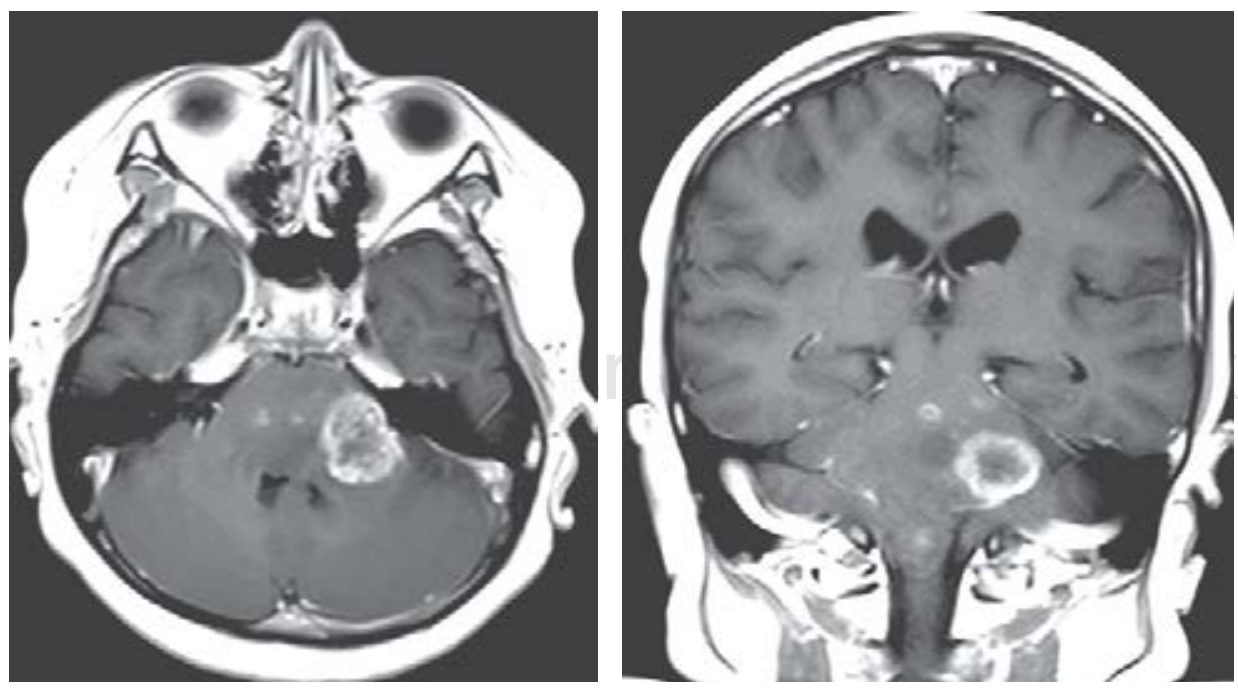

Figura 3.

IRM contrastada en corte axial y coronal mostrando una lesión tumoral a nivel del pedúnculo cerebeloso medio e involucro del tallo cerebral con reforzamiento intenso y componente necrótico en el interior. 
los casos en la IRM inicial en una serie, de los cuales $66 \%$ de los pacientes presentaba un patrón de anillo, sugiriendo necrosis en el centro. ${ }^{4,13} \mathrm{~A}$ pesar del hecho de que la ERM puede ser útil, es importante subrayar que en contraste con los niños, la imagen radiológica de glioma de tallo maligno es inespecífica. ${ }^{40}$ Estudios previos revelaron que los diagnósticos radiológicos preoperatorios son incorrectos en $10-25 \%$ de los casos en pacientes mayores de 20 años de edad que presentaban una lesión captante de contraste en el tallo. Una de esas series detectó una patología benigna no neoplásica en $17 \%$. Sin embargo, hay muchas causas de lesiones captantes de contraste en el tallo cerebral, especialmente enfermedades inflamatorias e infecciosas, así como otros tumores no malignos (por ejemplo, astrocitoma pilocítico) o malignos (por ejemplo, linfomas). ${ }^{41-47}$ De tal modo que aunque se realice una adecuada evaluación sistémica, neurológica y radiológica completa puede llevar a un diagnóstico incorrecto, la biopsia debe considerarse cuando estas pruebas no son concluyentes. En esta situación, la tomografía por emisión de positrones (PET-Scan) usando FDG ([18F] fluorodesoxiglucosa) y MET [11C]-metionina) puede ser útil para aumentar el diagnóstico mediante la definición del blanco de la biopsia (Figura 3). $.48,49$

\section{Principios de tratamiento}

\section{Cirugía}

El riesgo de complicaciones graves de la biopsia estereotáctica ante la sospecha de glioma maligno de tallo es bajo (entre 1-5.6\%). ${ }^{41,42}$ En una serie que incluyó 71 pacientes que se sometieron a biopsia guiada por tomografía, el déficit neurológico permanente ocurrió en $1.4 \%$ (un paciente) y el empeoramiento transitorio en $5.6 \%$ (cuatro pacientes). ${ }^{41}$ En otra serie de 46 biopsias de IMR/CT-guiadas en una cohorte de adultos con lesiones de tallo cerebral (incluyendo todos los grupos), la morbilidad perioperatoria fue de $2.5 \%$ (un paciente) y no hubo morbilidad permanente ni muerte. ${ }^{29}$ En un metaanálisis realizado de 1,480 casos se concluyó la viabilidad y seguridad de tumores de tallo (que incluía todos los tipos histológicos) y se reportó $96.2 \%$ de éxito de diagnóstico, $7.8 \%$ de morbilidad global, $1.7 \%$ de morbilidad permanente y $0.9 \%$ de mortalidad. ${ }^{50}$ En la literatura, la morbilidad suele ser mínima y temporal, consiste en el deterioro neurológico transitorio o hidrocefalia obstructiva. La mortalidad operatoria es extremada- mente rara. ${ }^{29,30,41,42}$ En general, cuanto menor sea la lesión que se encuentre en el tallo cerebral, mayores son los riesgos involucrados.

La cirugía estereotáctica guiada por tomografía computarizada (TC), IRM y si es posible por tomografía por emisión de positrones (PET) del tallo cerebral parece ser segura, fiable y proporciona un diagnóstico histológico acertado que se estima en la literatura en torno a 95\%. ${ }^{41-44,51}$ Sin embargo, el método y los abordajes para una biopsia óptima siguen siendo un tema de debate. ${ }^{52}$

\section{Radioterapia}

El GTMRF es altamente resistente al tratamiento con radioterapia. En efecto, después de la radioterapia, sólo 13\% (2/15 pacientes) mostraron mejoría clínica y radiológica. ${ }^{4}$ La radiocirugía con Gamma Knife se ha reportado en algunos casos de glioma de tallo de alto grado, pero los datos publicados son limitados. ${ }^{53}$

\section{Quimioterapia}

La quimioterapia se ha utilizado en ocasiones en este subgrupo de tumores, pero los datos son demasiado escasos para establecer alguna recomendación sobre este tema. Se desconoce la forma difusa intrínseca y a pesar de alentar los informes de casos, la administración concomitante y adyuvante de la temozolamida al momento del diagnóstico aporta el beneficio demostrado en glioblastomas supratentoriales. ${ }^{35}$ Ocasionalmente, los pacientes pueden responder a la quimioterapia por adelantado como se ilustra en el informe de un paciente con un glioblastoma confirmado por patología, que recibió TMZ sola (sin radioterapia) en $150-200 \mathrm{mg} / \mathrm{m}^{2}$ cada 28 días (un total de 17 ciclos) y mostró respuesta radiológica total y mejoría clínica con una supervivencia global de 37 meses. Estos casos son excepciones obvias. ${ }^{54}$

En una serie en la que se aplicó bevacizumab a un paciente con astrocitoma anaplásico grado III de la OMS, hubo mejoría en el KPS de 60 a 80, se redujo la dosis de dexametasona diaria hasta siete veces y en la IRM en T2 se observó disminución del tamaño de las lesiones hiperintensas y captantes de contraste. ${ }^{55}$

En la recaída se ha reportado la respuesta a la quimioterapia después de un fallo en la radioterapia en una minoría de los pacientes (aproximadamente 15\%), como se señaló con anterioridad. 

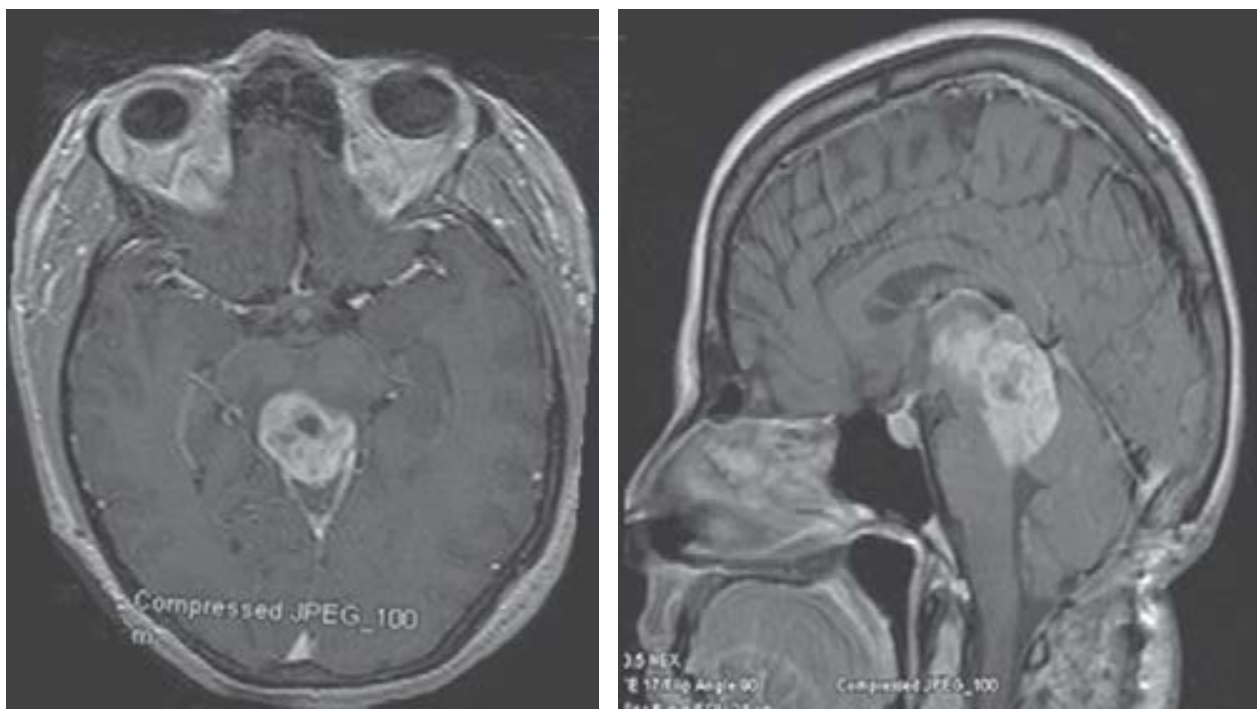

Figura 4.

IRM contrastada en corte axial y coronal con captación intensa del medio de contraste de localización tectal y extensión a la región pineal.

Es claro que se necesitan estudios prospectivos sobre este tema, así como la evaluación de nuevas terapias, como los fármacos antiangiogénicos.

\section{Pronóstico}

En contraste con los gliomas de bajo grado, en particular la forma intrínseca difusa, el pronóstico de los gliomas del tallo cerebral maligno es sombrío, con una supervivencia media de aproximadamente un año. ${ }^{7,9,12}$ Como los gliomas supratentoriales de alto grado suelen aparecer en edad avanzada (> 50 años), condicionan un factor pronóstico peyorativo. ${ }^{9}$

\section{Gliomas focales tectales de tallo}

\section{Epidemiología}

El glioma focal tectal es una entidad anatomo-clínica bien definida en los niños, comúnmente asociada a un resultado favorable. ${ }^{56,57}$ Los tumores se encuentran en la parte posterior del mesencéfalo, detrás del acueducto de Silvio sin evidente infiltración en los tejidos adyacentes. Los gliomas focales tectales constituyen un pequeño subgrupo en adultos ( $8 \%$ de los casos) y parecen idénticos al subtipo de la población pediátrica. ${ }^{7}$

\section{Patología}

En los pocos casos en los que se obtienen, los estudios histopatológicos revelan con mayor frecuencia oligoastrocitoma grado II. ${ }^{4}$ Sin embargo, también se han descrito algunos casos de astrocitomas pilocíticos, así como raros casos de glioblastoma. ${ }^{58}$

\section{Presentación clínica y radiológica}

Los tumores de la placa tectal suelen ser lesiones intrínsecas bien definidas. La ubicación periacueductal, la falta de captación del contraste y largos periodos de estabilidad son características clásicas de gliomas tectales focales. Las circunstancias clínicas que conducen al diagnóstico son variables y un hallazgo incidental es posible. Por lo general, los signos y síntomas progresivos de la hidrocefalia obstructiva anuncian la enfermedad y requieren derivación. ${ }^{7,46}$ Rara vez la hidrocefalia obstructiva es aguda y una hemorragia intracraneal constituye la primera manifestación, hallazgos neurológicos focales clínicos tales como la parálisis de nervios craneales (ej. III y IV) son menos frecuentes y pueden volver después de la normalización de la presión intracraneal (Figura 4). ${ }^{59,60}$

\section{Tratamiento y seguimiento}

Estas lesiones son consideradas como un subconjunto diferente de los tumores del tallo cerebral y se han asociado a una mayor media de supervivencia (superior a 10 años) después de la derivación ventrículo-peritoneal y en algunos casos radioterapia focal. ${ }^{55,56}$ Puesto que la simple observación después de la derivación puede ser una opción adecuada en niños, la decisión de tratar a los pacientes adultos con radioterapia también es 
cuestionable para algunos autores que proponen un enfoque conservador similar para éstos. ${ }^{4}$ Otra opción de tratamiento es braquiterapia estereotáctica con yodo125. En un estudio realizado en 47 pacientes a dosis de 50 a 65 Gy, la supervivencia libre de progresión fue de $81.6 \%$, no hubo mortalidad y dentro de los primeros 30 días después de la implantación $12.8 \%$ de los pacientes presentaron déficits neurológicos transitorios y $4.3 \%$ se deterioraron permanentemente. $19.1 \%$ presentaron recidiva tumoral, $23.4 \%$ respuesta completa, $29.8 \%$ respuesta parcial y $27.7 \%$ enfermedad estable. ${ }^{61}$

\section{Gliomas exofíticos de tallo}

El glioma exofítico captante de contraste, ya estudiado en niños (hasta 10\% de los casos) y que se asocia a un buen pronóstico, es muy poco frecuente en adultos, tal vez porque la mayoría de los gliomas exofíticos son astrocitomas pilocíticos. ${ }^{4,5}$ Sin embargo, también se ha visto un caso de glioblastoma cerebral pontocerebeloso en paciente de edad avanzada con mal pronóstico. ${ }^{62}$ En los adultos debe tenerse mucha precaución, ya que puede atribuirse una masa cerebral captante de contraste a este tipo de lesión benigna y los gliomas malignos pueden simularlos (Lee et al. ${ }^{63,64}$ Luetjens et al. $\left.{ }^{64,65}\right)$, destacando la importancia de la confirmación histológica. Sin embargo, si existe una lesión focal se asocia a un diagnóstico histopatológico favorable, existiendo buena correlación entre los hallazgos de imagen e histopatológicos. ${ }^{66}$

La resección quirúrgica se recomienda en algunos casos, tales como tumores exofíticos dorsales que sobresalen en el cuarto ventrículo. En una serie de siete pacientes con ganglioma grado I o II de la OMS se les trató sólo con cirugía logrando resecciones parciales y totales sin tratamiento complementario. En el seguimiento hasta cinco años mostraron estabilidad o mejoría de los síntomas y en IRM se observaron sólo lesiones residuales sin cambios de progresión o recurrencia. ${ }^{67}$

El avance en las técnicas neuroquirúrgicas (en particular el uso de la ecografía intraoperatoria, mapeo cerebral intraoperatorio, el uso de tensor de difusión (DTI), monitoreo neurofisiológico intraoperatorio, técnicas de reconstrucción por computadora y neuronavegación) han facilitado la selección del abordaje quirúrgico, la resección parcial de tumores con mayor seguridad, así como la eliminación total de tumores considerados como inoperables o incluso descompresión en algunos casos. ${ }^{23,59,68-70}$ La cirugía guiada por esterotaxia puede ser un método rápido y seguro para la evacuación del contenido de quistes que aporte un beneficio neurológico en la mayoría casos. ${ }^{18}$

\section{ConCLusión}

A pesar de la mejor comprensión de los gliomas de tallo cerebral, el reto principal sigue siendo un mejor conocimiento de estos tumores, en particular su clasificación y la mejora de estrategias de tratamiento que han logrado pocos avances en las últimas dos décadas. Esto requerirá avances en la comprensión de la biología de estos tumores y enfoques innovadores, posiblemente basados en el análisis del perfil histológico molecular. Los modelos animales experimentales de gliomas de tallo cerebral y estudios preclínicos en humanos son necesarios para investigar nuevos fármacos, como los inhibidores de transducción de señales que están en estudio para niños y posteriormente podrían beneficiar a pacientes adultos. ${ }^{71-73}$ Por último, los criterios para evaluar la respuesta del tumor durante la terapia de gliomas de tallo cerebral siguen siendo poco claros y requieren nuevos enfoques.

\section{BibLIOGRAFÍA}

1. Barkovich AJ, Krischer J, Kun LE, Packer R, Zimmerman RA, Freeman CR et al. Brain stem gliomas: a classification system based on magnetic resonance imaging. Pediatr Neurosurg. 1990-1991; 16 (2): 73-83.

2. Donaldson SS, Laningham F, Fisher PG. Advances toward an understanding of brainstem gliomas. J Clin Oncol. 2006; 24 (8): 1266-11272.

3. Fischbein NJ, Prados MD, Wara W, Russo C, Edwards MS, Barkovich AJ. Radiologic classification of brain stem tumors: correlation of magnetic resonance imaging appearance with clinical outcome. Pediatr Neurosurg. 1996; 24 (1): 9-23.

4. Guillamo JS, Monjour A, Taillandier L, Devaux B, Varlet P, HaieMeder $\mathrm{C}$ et al. Brainstem gliomas in adults: prognostic factors and classification. Brain. 2001; 124 (Pt 12): 2528-2539.

5. Landolfi JC, Thaler HT, DeAngelis LM. Adult brainstem gliomas. Neurology. 1998; 51 (4): 1136-1139.

6. Reyes-Botero G, Mokhtari K, Martin-Duverneuil N, Delattre JY, Laigle-Donadey F. Adult brainstem gliomas. Oncologist. 2012; 17 (3): 388-397.

7. Mohanty A. Role of surgery in brainstem gliomas. Neurol India. 2009; 57 (3): 231-232.

8. Salmaggi A, Fariselli L, Milanesi I, Lamperti E, Silvani A, Bizzi A et al. Natural history and management of brainstem gliomas in adults. A retrospective Italian study. J Neurol. 2008; 255 (2): 171-177.

9. Rineer J, Schreiber D, Choi K, Rotman M. Characterization and outcomes of infratentorial malignant glioma: a population-based study using the Surveillance Epidemiology and End-Results database. Radiother Oncol. 2010; 95 (3): 321-226.

10. Kaufman B. Brainstem gliomas. In: Berger MS, Prados MD. Textbook of neuro-oncology. Philadelphia, PA: Saunders; 2004. 
11. Grimm SA, Chamberlain MC. Brainstem glioma: a review. Curr Neurol Neurosci Rep. 2013; 13 (5): 346.

12. Kesari S, Kim RS, Markos V, Drappatz J, Wen PY, Pruitt AA. Prognostic factors in adult brainstem gliomas: a multicenter, retrospective analysis of 101 cases. J Neurooncol. 2008; 88 (2): 175-183.

13. Guillamo JS, Doz F, Delattre JY. Brain stem gliomas. Curr Opin Neurol. 2001; 14 (6): 711-715.

14. Hu J, Western S, Kesari S. Brainstem glioma in adults. Front Oncol. 2016; 6: 180.

15. Selvapandian S, Rajshekhar V, Chandy MJ. Brainstem glioma: comparative study of clinico-radiological presentation, pathology and outcome in children and adults. Acta Neurochir (Wien). 1999; 141 (7): 721-726; discussion 726-727.

16. Marin-Sanabria EA, Kobayashi N, Miyake S, Kohmura E. Snoring associated with Ondine's curse in a patient with brainstem glioma. J Clin Neurosci. 2006; 13 (3): 370-373.

17. Gaviani P, Gonzalez RG, Zhu JJ, Batchelor TT, Henson JW. Central neurogenic hyperventilation and lactate production in brainstem glioma. Neurology. 2005; 64 (1): 166-167.

18. Saka E, Ozkaynak S, Tuncer R. Tongue tremor in brainstem pilocytic astrocytoma. J Clin Neurosci. 2006; 13 (4): 503-506.

19. Yoshimura M, Yamamoto T, Iso-o N, Imafuku I, Momose T, Shirouzu I et al. Hemiparkinsonism associated with a mesencephalic tumor. J Neurol Sci. 2002; 197 (1-2): 89-92.

20. Lim EC, Wilder-Smith EP, Chong JL, Wong MC. Seeing the light: brainstem glioma causing visual auras and migraine. Cephalalgia. 2005; 25 (2): 154-156.

21. Ikeda K, Baba S, Iwasaki Y. Pseudomyasthenia in a patient with pontine glioma. Clin Neurol Neurosurg. 2006; 108 (8): 809-810.

22. Alvarez-Linera J. Magnetic resonance techniques for the brainstem. Semin Ultrasound CT MR. 2010; 31 (3): 230-245.

23. Panigrahy A, Nelson MD Jr, Finlay JL, Sposto R, Krieger MD, Gilles FH et al. Metabolism of diffuse intrinsic brainstem gliomas in children. Neuro Oncol. 2008; 10 (1): 32-44.

24. Porto L, Hattingen E, Pilatus U, Kieslich M, Yan B, Schwabe D et al. Proton magnetic resonance spectroscopy in childhood brainstem lesions. Childs Nerv Syst. 2007; 23 (3): 305-314.

25. Giussani C, Poliakov A, Ferri RT, Plawner LL, Browd SR, Shaw DW et al. DTI fiber tracking to differentiate demyelinating diseases from diffuse brain stem glioma. Neuroimage. 2010; 52 (1): 217-223.

26. Piette C, Munaut C, Foidart JM, Deprez M. Treating gliomas with glucocorticoids: from bedside to bench. Acta Neuropathol. 2006; 112 (6): 651-664.

27. Mursch K, Halatsch ME, Markakis E, Behnke-Mursch J. Intrinsic brainstem tumours in adults: results of microneurosurgical treatment of 16 consecutive patients. Br J Neurosurg. 2005; 19 (2): 128-136.

28. Tanaka S, Kobayashi I, Utsuki S, Iwamoto K, Takanashi J. Biopsy of brain stem glioma using motor-evoked potential mapping by direct peduncular stimulation and individual adjuvant therapy. Case report. Neurol Med Chir (Tokyo). 2005; 45 (1): 49-55.

29. Rachinger W, Grau S, Holtmannspötter M, Herms J, Tonn JC, Kreth FW. Serial stereotactic biopsy of brainstem lesions in adults improves diagnostic accuracy compared with MRI only. J Neurol Neurosurg Psychiatry. 2009; 80 (10): 1134-1139.

30. Reithmeier T, Kuzeawu A, Berger H, Trippel M, Nikkhah G. Adult brainstem gliomas: a retrospective analysis of 110 cases within the German Glioma Network 60th Annual Meeting of the German Society of Neurosurgery (DGNC); May 24-27, 2009; Münster, Germany.
31. Dellaretti M, Touzet G, Reyns N, Dubois F, Gusmão S, Pereira JL et al. Correlation between magnetic resonance imaging findings and histological diagnosis of intrinsic brainstem lesions in adults. Neuro Oncol. 2012; 14 (3): 381-385.

32. Elhammady MS, Teo C. Surgical management of adult intrinsic brainstem tumors. Neurosurgery. 2013; 60 Suppl 1: 131-138.

33. Fang $\mathrm{HH}, \mathrm{Nie} \mathrm{Q}$, Kang JB, Li FM, Cai CL. Safety and efficacy of three-dimensional conformal radiotherapy combined with temozolomide in treatment of diffuse brainstem gliomas. Zhonghua Zhong Liu Za Zhi. 2011; 33 (9): 707-709.

34. Torcuator R, Zuniga R, Loutfi R, Mikkelsen T. Bevacizumab and irinotecan treatment for progressive diffuse brainstem glioma: case report. J Neurooncol. 2009; 93 (3): 409-412.

35. Raza S, Donach M. Bevacizumab in adult malignant brainstem gliomas. J Neurooncol. 2009; 95 (2): 299-300.

36. Gururangan S, Chi SN, Young-Poussaint T, Onar-Thomas A, Gilbertson RJ, Vajapeyam S et al. Lack of efficacy of bevacizumab plus irinotecan in children with recurrent malignant glioma and diffuse brainstem glioma: a Pediatric Brain Tumor Consortium study. J Clin Oncol. 2010; 28 (18): 3069-3075.

37. Dellaretti M, Reyns N, Touzet G, Dubois F, Gusmão S, Pereira JL et al. Diffuse brainstem glioma: prognostic factors. J Neurosurg. 2012; 117 (5): 810-814.

38. Stark AM, Maslehaty H, Hugo HH, Mahvash M, Mehdorn HM. Glioblastoma of the cerebellum and brainstem. J Clin Neurosci. 2010; 17 (10): 1248-1251.

39. Babu R, Kranz PG, Karikari IO, Friedman AH, Adamson C. Clinical characteristics and treatment of malignant brainstem gliomas in elderly patients. J Clin Neurosci. 2013; 20 (10): 1382-1386.

40. Kwon JW, Kim IO, Cheon JE, Kim WS, Moon SG, Kim TJ et al. Paediatric brain-stem gliomas: MRI, FDG-PET and histological grading correlation. Pediatr Radiol. 2006; 36 (9): 959-964.

41. Rajshekhar V, Chandy MJ. Computerized tomography-guided stereotactic surgery for brainstem masses: a risk-benefit analysis in 71 patients. J Neurosurg. 1995; 82 (6): 976-981.

42. Boviatsis EJ, Kouyialis AT, Stranjalis G, Korfias S, Sakas DE. CTguided stereotactic biopsies of brain stem lesions: personal experience and literature review. Neurol Sci. 2003; 24 (3): 97-102.

43. Friedman WA, Sceats DJ Jr, Nestok BR, Ballinger WE Jr. The incidence of unexpected pathological findings in an imageguided biopsy series: a review of 100 consecutive cases. Neurosurgery. 1989; 25 (2): 180-184.

44. Gonçalves-Ferreira AJ, Herculano-Carvalho M, Pimentel J. Stereotactic biopsies of focal brainstem lesions. Surg Neurol. 2003; 60 (4): 311-320; discussion 320.

45. Swinson BM, Friedman WA, Yachnis AT. Pontine atypical neurocytoma: case report. Neurosurgery. 2006; 58 (5): E990; discussion E990.

46. Shenoy SN, Raja A. Cystic trochlear nerve neurinoma mimicking intrinsic brainstem tumour. Br J Neurosurg. 2004; 18 (2): 183-186.

47. Yousry I, Muacevic A, Olteanu-Nerbe V, Naidich TP, Yousry TA. Exophytic pilocytic astrocytoma of the brain stem in an adult with encasement of the caudal cranial nerve complex (IX-XII): presurgical anatomical neuroimaging using MRI. Eur Radiol. 2004; 14 (7): 1169-1173.

48. Massager N, David P, Goldman S, Pirotte B, Wikler D, Salmon I et al. Combined magnetic resonance imaging- and positron emission tomography-guided stereotactic biopsy in brainstem mass lesions: diagnostic yield in a series of 30 patients. J Neurosurg. 2000; 93 (6): 951-957.

49. Pirotte BJ, Lubansu A, Massager N, Wikler D, Van Bogaert P, Levivier $\mathrm{M}$ et al. Clinical impact of integrating positron emission 
tomography during surgery in 85 children with brain tumors. J Neurosurg Pediatr. 2010; 5 (5): 486-499.

50. Kickingereder P, Willeit P, Simon T, Ruge MI. Diagnostic value and safety of stereotactic biopsy for brainstem tumors: a systematic review and meta-analysis of 1480 cases. Neurosurgery. 2013; 72 (6): 873-881; discussion 882; quiz 882.

51. Abernathey CD, Camacho A, Kelly PJ. Stereotaxic suboccipital transcerebellar biopsy of pontine mass lesions. J Neurosurg. 1989; 70 (2): 195-200.

52. Kratimenos GP, Thomas DG. The role of image-directed biopsy in the diagnosis and management of brainstem lesions. Br J Neurosurg. 1993; 7 (2): 155-164.

53. Fuchs I, Kreil W, Sutter B, Papaethymiou G, Pendl G. Gamma Knife radiosurgery of brainstem gliomas. Acta Neurochir Suppl. 2002; 84: 85-90.

54. Zhang C, Yao Y, Wang Y, Chen Z, Wu J, Mao Y, Zhou L. Temozolomide for adult brain stem glioblastoma: case report of a long-term survivor. Int J Neurosci. 2010; 120 (12): 787-791.

55. Reithmeier T, Lopez WO, Spehl TS, Nguyen T, Mader I, Nikkhah $\mathrm{G}$ et al. Bevacizumab as salvage therapy for progressive brain stem gliomas. Clin Neurol Neurosurg. 2013; 115 (2): 165-169.

56. Hamilton MG, Lauryssen C, Hagen N. Focal midbrain glioma: long term survival in a cohort of 16 patients and the implications for management. Can J Neurol Sci. 1996; 23 (3): 204-207.

57. Bowers DC, Georgiades C, Aronson LJ, Carson BS, Weingart JD, Wharam MD et al. Tectal gliomas: natural history of an indolent lesion in pediatric patients. Pediatr Neurosurg. 2000; 32 (1): 24-29.

58. Chaddad Neto F, Lopes A, Alberto-Filho M, Catanoce A, Joaquim AF, Oliveira Ed. Tectal glioblastoma. Arq Neuropsiquiatr. 2007; 65 (4A): 996-999.

59. Dağlioğlu E, Cataltepe O, Akalan N. Tectal gliomas in children: the implications for natural history and management strategy. Pediatr Neurosurg. 2003; 38 (5): 223-231.

60. Oka F, Yamashita Y, Kumabe T, Tominaga T. Total resection of a hemorrhagic tectal pilocytic astrocytoma--case report. Neurol Med Chir (Tokyo). 2007; 47 (5): 219-221.

61. Ruge MI, Kickingereder P, Simon T, Treuer H, Sturm V. Stereotactic iodine-125 brachytherapy for treatment of inoperable focal brainstem gliomas of WHO grades I and II: feasibility and long-term outcome. J Neurooncol. 2012; 109 (2): 273-283.

62. Salunke P, Sura S, Tewari MK, Gupta K, Khandelwal NK. An exophytic brain stem glioblastoma in an elderly presenting as a cerebellopontine angle syndrome. Br J Neurosurg. 2012; 26 (1): 96-98.

63. Lee TT, Galarza M, Petito CK, Heros RC. Exophytic malignant brainstem mixed glioma in an adult: a case report. J Neurooncol. 1998; 37 (2): 123-129.
64. Swaroop GR, Whittle IR. Exophytic pontine glioblastoma mimicking acoustic neuroma. J Neurosurg Sci. 1997; 41 (4): 409-411.

65. Luetjens G, Mirzayan MJ, Brandis A, Krauss JK. Exophytic giant cell glioblastoma of the medulla oblongata. J Neurosurg. 2009; 110 (3): 589-593.

66. Yin L, Zhang L. Correlation between MRI findings and histological diagnosis of brainstem glioma. Can J Neurol Sci. 2013; 40 (3): 348-354.

67. Zhang S, Wang X, Liu X, Ju Y, Hui X. Brainstem gangliogliomas: a retrospective series. J Neurosurg. 2013; 118 (4): 884-888.

68. Liu L, Hui XH, Zhang S, Fang Y, Zhang YK, Xie XP. Microsurgical management of adult brainstem gliomas under the DTI and intraoperative nuclei mapping: a retrospective analysis of 19 cases. Sichuan Da Xue Xue Bao Yi Xue Ban. 2013; 44 (3): 489493.

69. Royo A1, Utrilla C, Carceller F. Surgical management of brainstem-expanding lesions: the role of neuroimaging. Semin Ultrasound CT MR. 2013; 34 (2): 153-173.

70. Recinos PF, Rahmathulla G, Jallo GI, Recinos VR. Brainstem tumors: current management and future directions. IJNS. 2012; 1 (1): 48-53.

71. Fouladi M, Nicholson HS, Zhou T, Laningham F, Helton KJ, Holmes $\mathrm{E}$ et al. A phase II study of the farnesyl transferase inhibitor, tipifarnib, in children with recurrent or progressive high-grade glioma, medulloblastoma/primitive neuroectodermal tumor, or brainstem glioma: a Children's Oncology Group study. Cancer. 2007; 110 (11): 2535-2541.

72. Pollack IF, Jakacki RI, Blaney SM, Hancock ML, Kieran MW, Phillips P et al. Phase I trial of imatinib in children with newly diagnosed brainstem and recurrent malignant gliomas: a Pediatric Brain Tumor Consortium report. Neuro Oncol. 2007; 9 (2): 145-160.

73. Pollack IF, Stewart CF, Kocak M, Poussaint TY, Broniscer A, Banerjee A et al. A phase II study of gefitinib and irradiation in children with newly diagnosed brainstem gliomas: a report from the Pediatric Brain Tumor Consortium. Neuro Oncol. 2011; 13 (3): 290-297.
Correspondencia:

Alberto González-Aguilar

Insurgentes Sur Núm. 3877,

Col. La Fama, 14269,

Del. Tlalpan, Ciudad de México.

Teléfono: 56063822, ext. 2120

E-mail: albertogonzalez@neurocirugia-innn.com 